\title{
Importance of surface oxygen vacancies for ultrafast hot carrier relaxation and transport in $\mathrm{Cu}_{2} \mathrm{O}$
}

\author{
Chiara Ricca, ${ }^{1,2}$ Lisa Grad, ${ }^{3}$ Matthias Hengsberger $\odot,{ }^{3}$ Jürg Osterwalder $\odot,{ }^{3}$ and Ulrich Aschauer $\oplus^{1,2, *}$ \\ ${ }^{1}$ Department of Chemistry and Biochemistry, University of Bern, Freiestrasse 3, CH-3012 Bern, Switzerland \\ ${ }^{2}$ National Centre for Computational Design and Discovery of Novel Materials (MARVEL), CH-1015 Lausanne, Switzerland \\ ${ }^{3}$ Department of Physics, University of Zurich, Winterthurerstrasse 190, CH-8057 Zurich, Switzerland
}

(Received 17 March 2021; revised 24 August 2021; accepted 3 December 2021; published 27 December 2021)

\begin{abstract}
$\mathrm{Cu}_{2} \mathrm{O}$ has appealing properties as an electrode for photoelectrochemical water splitting, yet its practical performance is severely limited by inefficient charge extraction at the interface. Using hybrid DFT calculations, we investigate carrier capture processes by oxygen vacancies $\left(V_{\mathrm{O}}\right)$ in the experimentally observed $(\sqrt{3} \times \sqrt{3}) R 30^{\circ}$ reconstruction of the dominant (111) surface. Our results show that these $V_{\mathrm{O}}$ are doubly ionized and that associated defects states strongly suppress electron transport. In particular, the excited electronic state of a singly charged $V_{\mathrm{O}}$ plays a crucial role in the nonradiative electron capture process with a capture coefficient of about $10^{-9} \mathrm{~cm}^{3} / \mathrm{s}$ and a lifetime of $0.04 \mathrm{ps}$, explaining the experimentally observed ultrafast carrier relaxation. These results highlight that engineering the surface $V_{\mathrm{O}}$ chemistry will be a crucial step in optimizing $\mathrm{Cu}_{2} \mathrm{O}$ for photoelectrode applications.
\end{abstract}

DOI: 10.1103/PhysRevResearch.3.043219

\section{INTRODUCTION}

Cuprous oxide $\left(\mathrm{Cu}_{2} \mathrm{O}\right)$ is a promising material for a variety of industrial applications due to its small direct band gap, its high absorbance, the abundance and nontoxicity of its constituent elements, and the large flexibility and low cost of $\mathrm{Cu}_{2} \mathrm{O}$-based thin-film preparation methods [1]. In particular, cuprous oxide has recently attracted much attention as an electrode material for photoelectrochemical water splitting with efficient light absorption, high positive onset voltage, and high photocurrent density [2,3]. However, the electrode performance is highly sensitive to the presence of defect states generally appearing within the semiconductor band gap $[4,5]$. Such defect states may trap excited charge carriers resulting in a reduction of the generated photovoltage and photocurrent.

In bulk $\mathrm{Cu}_{2} \mathrm{O}$ both copper and oxygen vacancies occur, copper vacancies being more abundant and generally leading to $p$-type doping. The (111) facet is the experimentally most studied $\mathrm{Cu}_{2} \mathrm{O}$ surface and two ordered surface structures were identified. The first corresponds to the ideal O-terminated and stoichiometric surface with $(1 \times 1)$ periodicity. The second is a reconstructed $(\sqrt{3} \times \sqrt{3}) R 30^{\circ}$ surface with only $2 / 3$ of the $\mathrm{O}$ ions in the outermost surface layer [6-8]. The exact termination, stoichiometry, and atomic structure of the reconstructed surface have been studied by experiment [6-8] and

\footnotetext{
*ulrich.aschauer@dcb.unibe.ch

Published by the American Physical Society under the terms of the Creative Commons Attribution 4.0 International license. Further distribution of this work must maintain attribution to the author(s) and the published article's title, journal citation, and DOI.
}

theory [9-13], associating it most likely with a $1 / 3$ monolayer of ordered oxygen vacancies $\left(V_{\mathrm{O}}\right)$ [6-8].

Recently, it was suggested that these surface $V_{O}$ dominantly affect the photocatalytic performance in $\mathrm{Cu}_{2} \mathrm{O}$-based materials under oxygen poor conditions [5]. Comparing electron dynamics at stoichiometric and oxygen deficient $\mathrm{Cu}_{2} \mathrm{O}-(111)$ surfaces observed via time-resolved two-photon photoemission (2PPE) indicates that surface rather than bulk defects limit the photovoltage. Excited electrons can drift within the conduction band to the stoichiometric $\mathrm{Cu}_{2} \mathrm{O}-(111)$ surface where they create an energetic electron population that decays with a characteristic time of $10 \mathrm{ps}$. On the oxygen deficient $(\sqrt{3} \times \sqrt{3}) R 30^{\circ}-(111)$ surface, however, no indication of electrons occupying the conduction band was found, but instead electrons are trapped, within $1 \mathrm{ps}$, by low-lying defect states with no further noticeable change of the electron population over a timescale of hundreds of ps. This indicates that $V_{\mathrm{O}}$ associated with the reconstruction likely trap carriers.

Unfortunately, previous experimental $[6-8,14]$ and theoretical [15-19] work reports contradictory results for $V_{O}$ in $\mathrm{Cu}_{2} \mathrm{O}$, especially in terms of the thermodynamic stability of different charge states, the position of the corresponding defect levels in the band gap, and consequently their ability to act as efficient carrier traps [see Supplemental Material (SM) [20] Sec. S3]. In addition, these theoretical studies are restricted to the bulk and the experimentally relevant carrier capture at surface defects has never been computationally studied.

Here we investigate the stability and electronic properties of $V_{\mathrm{O}}$ and the electron and hole trapping processes by these defects at the $\mathrm{Cu}_{2} \mathrm{O}$ surface by combining density functional theory (DFT) calculations and photoemission experiments. Accurately computing the electronic properties of $\mathrm{Cu}_{2} \mathrm{O}$ is a challenging task, due to the peculiar properties of this oxide: a mainly ionic semiconductor with closed shell $\mathrm{Cu}^{+}$ions and a higher coordination of $\mathrm{O}$ than $\mathrm{Cu}$ atoms, which leads to 
large cohesive energies. Semilocal DFT fails to predict the semiconducting character $[1,17], \mathrm{DFT}+U$ approaches also failing to open the band gap due to the fully occupied $\mathrm{Cu}$ $3 d^{10}$ states. All our calculations have hence been performed using hybrid functionals, which can successfully reproduce the band structure of $\mathrm{Cu}_{2} \mathrm{O}[18,21]$, but are rather expensive for applications in solid-state chemistry, especially with plane-wave-based codes and for surface/defect calculations that require large supercells. By comparing computed defect levels and electron capture coefficients with experimental results, we reach a deep understanding of the carrier dynamics and factors limiting photoelectrode performance. In particular, our results demonstrate that the defect states associated with the reconstructed $\mathrm{Cu}_{2} \mathrm{O}$-(111) surface are due to an ordered arrangement of doubly ionized $V_{\mathrm{O}}$, which effectively trap electrons and strongly suppress electron transport. This indicates that engineering the surface defect chemistry of $\mathrm{Cu}_{2} \mathrm{O}$-based photoelectrodes is crucial to enhance their performance.

\section{METHODS}

All density functional theory calculations were performed with the Vienna $A b$-initio Simulation Package (VASP) [22-25]. The Heyd-Scuseria-Ernzerhof (HSE) [26,27] hybrid exchange-correlation functional was employed together with the default VASP $\mathrm{Cu}$ and $\mathrm{O}$ projector augmented wave (PAW) potentials $[28,29]$ with $\mathrm{Cu}(4 s, 3 d)$ and $\mathrm{O}(2 s, 2 p)$ valence configurations. Wave functions were expanded in plane waves up to a kinetic energy of $500 \mathrm{eV}$. This setup provides an excellent agreement with experiment (see SM [20] Secs. S1A and S2).

Surface calculations were performed using a $(\sqrt{3} \times$ $\sqrt{3}) R 30^{\circ}$ supercell of the ideal O-terminated (111) surface from which one surface $\mathrm{O}$ atom was removed, corresponding to a $1 / 3 V_{\mathrm{O}}$ monolayer. The slab model consists of five O-Cu-O trilayers with a $10-\AA$ vacuum gap to prevent interactions between periodic images along the surface normal. The Brillouin zone of this slab model was sampled using a $3 \times 3 \times 1$ k-point mesh, resulting in defect transitions levels within $0.04 \mathrm{eV}$ of those obtained with a denser $4 \times 4 \times 1$ mesh. Different charge states for the $V_{\mathrm{O}}$ were studied by adjusting the number of electrons and by applying a neutralizing background charge, as required by calculations under periodic-boundary conditions to avoid a divergence of the electrostatic potential. Atomic positions were optimized while keeping the lattice vectors fixed at optimized values of the nondefective system. Additionally, the atoms in the bottom two trilayers were kept fixed at bulk positions. Structural relaxations were performed until forces converged below $10^{-3} \mathrm{eV} / \AA$.

The formation energy of a $V_{\mathrm{O}}$ in a charge state $q\left(E_{f, V_{\mathrm{O}}} q\right)$ was calculated as described in Ref. [30]:

$$
\begin{aligned}
E_{f, V_{\mathrm{O}} q}= & E_{\mathrm{tot}, V_{\mathrm{O}} q}-E_{\mathrm{tot}, \text { stoich }}+n_{\mathrm{O}} \mu_{\mathrm{O}} \\
& +q\left[E_{\mathrm{VBM}}+E_{\mathrm{F}}\right]+E_{\mathrm{corr}},
\end{aligned}
$$

where $E_{\mathrm{tot}, V_{0}}$ and $E_{\text {tot,stoich }}$ are the total energies of the defective and stoichiometric supercells, respectively, and $E_{\mathrm{F}}$ is the Fermi energy relative to the valence band maximum $\left(E_{\mathrm{VBM}}\right)$ of the stoichiometric cell, which can assume values within the band gap $E_{\mathrm{g}}\left(0 \leqslant E_{\mathrm{F}} \leqslant E_{\mathrm{g}}\right)$ of the nondefective structure. The third term includes the number of removed $\mathrm{O}$ atoms $\left(n_{\mathrm{O}}<0\right)$ and the oxygen chemical potential $\mu_{\mathrm{O}}$. Results are reported for oxygen-poor conditions: $\mu_{O}=\frac{1}{2} \mu_{\mathrm{O}_{2}}+$ $\Delta \mu_{\mathrm{O}}$, where $\mu_{\mathrm{O}_{2}}$ is the energy of the $\mathrm{O}_{2}$ molecule and $\Delta \mu_{\mathrm{O}}=$ $-1.55 \mathrm{eV}$ (see SM [20] Sec. S1B for details). Last, $E_{\text {corr }}$ is a corrective term necessary to align the electrostatic potentials of the charged defective and the neutral stoichiometric cell obtained by averaging the electrostatic potential in spheres around atomic sites located far from the defect [31]. We note that correction schemes specifically tailored for slab setups [32] would, in principle, be better suited, resulting in our case however in unphysical values of some of the parameters. No further finite-size corrections were applied since the defect concentration we simulate is realistic for this surface.

The thermodynamic transition level $\epsilon\left(q_{1} / q_{2}\right)$ for $V_{\mathrm{O}}$ defects with charge states $q_{1}$ and $q_{2}$ was computed as the Fermi level for which the formation energies of the two charge states are equal [30]:

$$
\epsilon\left(q_{1} / q_{2}\right)=\frac{E_{f, V_{\mathrm{O}} q_{1}}\left(E_{\mathrm{F}}=0\right)-E_{f, V_{\mathrm{O}} q_{2}}\left(E_{\mathrm{F}}=0\right)}{q_{2}-q_{1}},
$$

where $E_{f, V_{\mathrm{O}} q}$ is the formation energy of a $V_{\mathrm{O}}$ in a charge state $q$ in its equilibrium structure when the Fermi level is at the valence-band maximum $\left(E_{\mathrm{F}}=0\right)$. For Fermi-level positions below $\epsilon\left(q_{1} / q_{2}\right)$, the charge state $q_{1}$ is stable, while for Fermilevel positions above $\epsilon\left(q_{1} / q_{2}\right)$, the charge state $q_{2}$ is stable.

We computed surface energies via $a b$ initio atomistic thermodynamics, as shown for $\mathrm{Cu}_{2} \mathrm{O}$ surfaces in Ref. [10]:

$$
\gamma=\frac{1}{2 A}\left[E_{\mathrm{slab}}-N_{\mathrm{Cu}} \mu_{\mathrm{Cu}}-N_{\mathrm{O}} \mu_{\mathrm{O}}\right],
$$

where $E_{\text {slab }}$ is the total energy of the slab, $N_{\mathrm{Cu}} / N_{\mathrm{O}}$ are the number of $\mathrm{Cu} / \mathrm{O}$ atoms in the model, and $\mu_{\mathrm{Cu}} / \mu_{\mathrm{O}}$ are the $\mathrm{Cu} / \mathrm{O}$ chemical potentials. $A$ is the surface area and the $1 / 2$ factor accounts for the two surfaces contained in the slab. Result are reported for O-rich $\left(\Delta \mu_{\mathrm{O}}=-1.41 \mathrm{eV}\right.$ and $\left.\Delta \mu_{\mathrm{Cu}}=-0.07 \mathrm{eV}\right)$ and $\mathrm{O}$-poor conditions $\left(\Delta \mu_{\mathrm{Cu}}=0\right.$ and $\Delta \mu_{\mathrm{O}}=-1.55 \mathrm{eV}$; see SM [20] Sec. S1B for details).

We considered nonradiative carrier capture processes involving a defect and occurring via multiphonon emission $[33,34]$. Such processes can be described in DFT using the approach introduced by Alkauskas et al. [35,36] based on the static approximation [37]. This formalism was derived for the dilute defect limit, but due to the fairly localized defect states in this work still provides a good approximation even at higher defect concentrations. In addition we removed the restriction of harmonic vibrations by solving the one-dimensional (1D) Schrödinger equation for an anharmonic potential energy surface, following Ref. [38]. This method uses an effective one-dimensional configuration coordinate $Q$ to represent the phonon wave function of all vibrations coupling to the change of the defect geometry upon carrier capture. Within this approach, the capture coefficient is

$$
\begin{aligned}
C(T)= & s V \eta_{s p} g \frac{2 \pi}{\hbar} W_{i f}^{2} \sum_{m, n} w_{m}(T)\left|\left\langle\chi_{i m}|Q+\Delta Q| \chi_{f n}\right\rangle\right|^{2} \\
& \times \delta\left(\Delta E+\epsilon_{i m}-\epsilon_{f n}\right),
\end{aligned}
$$

where $V$ is the volume of the supercell, $\eta_{s p}$ accounts for spin-selection rules $\left(\eta_{s p}=1 / 2\right.$ when the initial state is a 
spin doublet and the final state is a spin singlet), $g$ is the degeneracy of the final state, $W_{i f}$ is the electron-phonon coupling matrix element of the initial and final states, $\Delta E$ is the energy difference between the two states, $\chi$ and $\epsilon$ are the phonon wave functions and eigenvalues, respectively, for the excited $(\mathrm{im})$ and ground $(f n)$ electronic states, while $w_{m}$ is the thermal occupation number of the excited vibrational state. $\delta$ is replaced with a Gaussian function of finite width of $0.14 \mathrm{eV}$, derived from the frequency of the effective vibration in the ground state as suggested in Ref. [39]. We note that for an anharmonic potential multiple Gaussian widths would result, but choose to work within the approximation of a single Gaussian of the above width. Matrix elements $W_{i f}$ are computed according to Ref. [35] using the PAWpySeed package [40] to obtain the wave-function overlap. The Sommerfeld factor $s$ was computed according to Refs. [41,42], requiring electron and hole effective masses, obtained by finite differences as implemented in the effective mass calculator (EMC) [43]. This factor has to be computed for charged defects as it accounts for the Coulomb interaction between the carrier and the charged defect that strongly affects the capture process. We stress that these interactions may not be well represented in supercell calculations that also use a neutralizing background to prevent divergence of the Coulomb potential. While customarily used in DFT calculations of defects to derive properties like defect formation energies or the thermodynamics transition levels, it remains to be shown if this approach could unphysically affect carrier capture, as discussed in Refs. [35,44]. Nonradiative capture coefficients were calculated with the CarrierCapture. $j 1$ package [45]. We note that convergence of the capture coefficients with respect to the cell size was not performed, since the defect concentration we investigate corresponds to the one in experiment. Additional information can be found in SM [20] Sec. S1C.

\section{RESULTS AND DISCUSSION}

\section{A. $(\sqrt{3} \times \sqrt{3}) R 30^{\circ}-(111) \mathrm{Cu}_{2} \mathrm{O}$ surface}

Figure 1 illustrates the slab model used to simulate the unreconstructed $(1 \times 1)$ and reconstructed $(\sqrt{3} \times \sqrt{3}) R 30^{\circ}$ (111) $\mathrm{Cu}_{2} \mathrm{O}$ surfaces within the $(\sqrt{3} \times \sqrt{3}) R 30^{\circ}$ supercell, where the $c$ axis is perpendicular to the surface plane. The unreconstructed surface consists of $\mathrm{O}-\mathrm{Cu}-\mathrm{O}$ trilayers, with each $\mathrm{Cu}$ layer sandwiched between two $\mathrm{O}$ layers. The topmost trilayer in the unreconstructed supercell contains four types of atoms: three coordinatively unsaturated $\mathrm{O}$ atoms $\left(\mathrm{O}_{\mathrm{CUS}}\right)$ in the first layer, three coordinatively unsaturated $\left(\mathrm{Cu}_{\mathrm{CUS}}\right)$ and nine coordinatively saturated $\mathrm{Cu}$ atoms $\left(\mathrm{Cu}_{\mathrm{CSA}}\right)$ in the second, and three coordinatively saturated $\mathrm{O}$ atoms $\left(\mathrm{O}_{\mathrm{CSA}}\right)$ in the third layer. Experiment suggests that the reconstructed oxygen-deficient surface, which we focus on in this work, is associated with a $1 / 3$ monolayer of charged surface $V_{\mathrm{O}_{\text {cus }}}$ forming ordered structures due to mutual electrostatic repulsion [6-8].

Surface energies computed for the ideal stoichiometric $\left(0.831 \mathrm{~J} / \mathrm{m}^{2}\right)$ and defective $\left(0.919 / 0.931 \mathrm{~J} / \mathrm{m}^{2}\right.$ under Opoor/rich conditions) surface are slightly higher (by about 0.2 and $0.1 \mathrm{~J} / \mathrm{m}^{2}$ respectively) compared to previous semilocal (a)
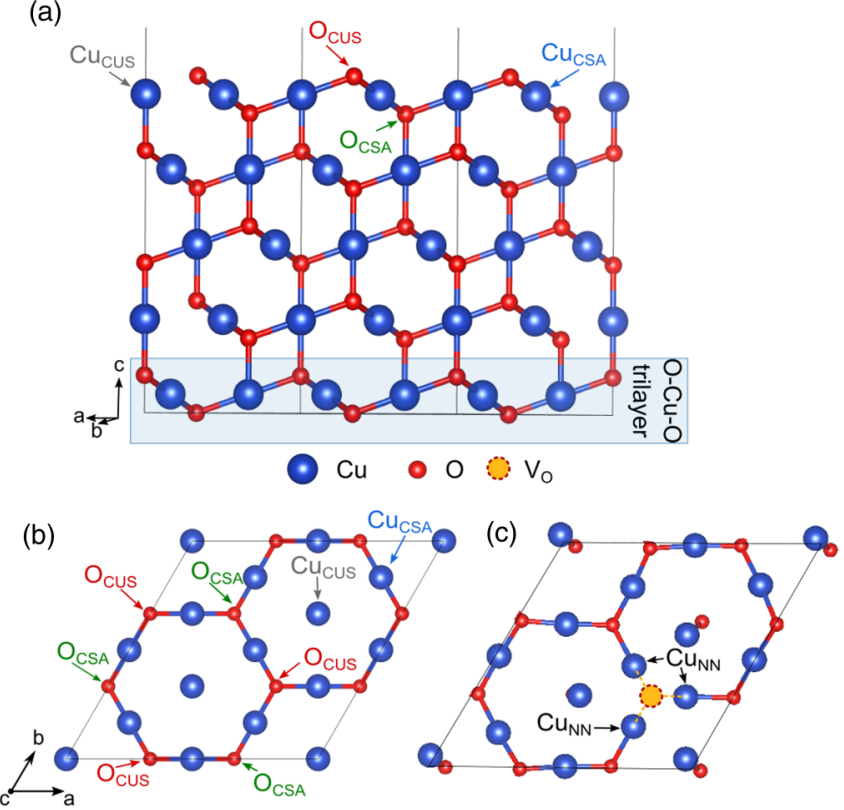

FIG. 1. (a) Lateral and (b) top view of the stoichiometric, unreconstructed $(1 \times 1)-(111) \mathrm{Cu}_{2} \mathrm{O}$ slab model within the $(\sqrt{3} \times$ $\sqrt{3}) R 30^{\circ}$ supercell. $\mathrm{O}_{\mathrm{CUS}}$ and $\mathrm{Cu}_{\mathrm{CUS}}$ are the coordinatively unsaturated $\mathrm{O}$ and $\mathrm{Cu}$ surface sites, respectively, while $\mathrm{O}_{\mathrm{CSA}}$ and $\mathrm{O}_{\mathrm{CSA}}$ indicate the coordinatively saturated $\mathrm{O}$ and $\mathrm{Cu}$ atoms. (c) Top view of the reconstructed $(\sqrt{3} \times \sqrt{3}) R 30^{\circ}-(111) \mathrm{Cu}_{2} \mathrm{O}$ slab model with one oxygen vacancy $\left(V_{\mathrm{O}}\right) . \mathrm{Cu}_{\mathrm{NN}}$ are $\mathrm{Cu}$ sites adjacent to $V_{\mathrm{O}}$.

DFT or DFT $+U$ results [10]. The qualitative picture is, however, in agreement, indicating the ideal surface to be slightly more stable than the reconstructed surface, regardless of the $\mathrm{O}$ chemical potential. This is also in line with the experimental conditions required to obtain the two surface structures: the ideal surface is generally obtained using milder conditions for the ion bombardment and with high-temperature annealing while the reconstructed surface is obtained for ion bombardment at higher kinetic energy and at lower annealing temperatures $[5,7,8]$.

The relaxed structure of the stoichiometric surface has $\mathrm{Cu}_{\mathrm{CUS}}-\mathrm{O}$ and $\mathrm{Cu}_{\mathrm{CSA}}-\mathrm{O}_{\mathrm{CUS}}$ bond lengths of about 1.90 and $1.81 \AA$, which are longer and shorter, respectively, compared to $\mathrm{Cu}-\mathrm{O}$ bonds in bulk $\mathrm{Cu}_{2} \mathrm{O}$ (see Table I). The formation of a neutral oxygen vacancy $\left(V_{\mathrm{O}}^{\bullet \bullet}\right.$ in Kröger-Vink notation [46]) due to removal of one $\mathrm{O}_{\text {Cus }}$ from the stoichiometric $(\sqrt{3} \times \sqrt{3}) R 30^{\circ}$ surface results in a vacant site surrounded

TABLE I. Interatomic distances on the reconstructed surface with neutral $\left(V_{\mathrm{O}}^{\bullet \bullet}\right)$, singly $\left(V_{\mathrm{O}}^{\bullet}\right)$ or doubly $\left(V_{\mathrm{O}}^{\mathrm{X}}\right)$ positively charged $V_{\mathrm{O}}$, compared to the stoichiometric surface and bulk $\mathrm{Cu}_{2} \mathrm{O}$. See Fig. 1 for atomic labels.

\begin{tabular}{lccccc}
\hline \hline Distance & $V_{\mathrm{O}}^{\bullet \bullet}(\AA)$ & $V_{\mathrm{O}}^{\bullet}(\AA)$ & $V_{\mathrm{O}}^{\mathrm{X}}(\AA)$ & Ideal $(\AA)$ & Bulk $(\AA)$ \\
\hline $\mathrm{Cu}_{\mathrm{NN}}-\mathrm{Cu}_{\mathrm{NN}}$ & 2.47 & 2.47 & 2.47 & 3.01 & 3.01 \\
$\mathrm{Cu}_{\mathrm{NN}}-\mathrm{O}_{\mathrm{CSA}}$ & 1.93 & 1.94 & 1.94 & 1.86 & 1.85 \\
$\mathrm{Cu}_{\mathrm{CSA}}-\mathrm{O}_{\mathrm{CUS}}$ & & & & 1.81 & 1.85 \\
$\mathrm{Cu}_{\mathrm{CUS}}-\mathrm{O}_{\mathrm{CSA}}$ & 1.92 & 1.92 & 1.92 & 1.90 & 1.85 \\
$\mathrm{Cu}_{\mathrm{CUS}}-\mathrm{Cu}_{\mathrm{NN}}$ & 2.54 & 2.64 & 2.58 & 3.01 & 3.01 \\
\hline \hline
\end{tabular}


by three singly coordinated $\mathrm{Cu}$ ions $\left[\mathrm{Cu}_{\mathrm{NN}}\right.$; see Fig. 1(c)] and leads to large structural relaxations: $\mathrm{Cu}_{\mathrm{NN}}$ move towards the $V_{\mathrm{O}}^{\bullet \bullet}$, forming a cluster of $\mathrm{Cu}$ ions with $\mathrm{Cu}_{\mathrm{NN}}-\mathrm{Cu}_{\mathrm{NN}}$ distances of about $2.47 \AA$ and at the same time, the $\mathrm{Cu}_{\mathrm{CUS}}$ ions also move towards the defect, resulting in $\mathrm{Cu}_{\mathrm{CUS}}-\mathrm{Cu}_{\mathrm{NN}}$ distances of about $2.54 \AA$, in both cases much shorter than the $\mathrm{Cu}-\mathrm{Cu}$ bond lengths in the bulk (3.01 $\AA$; see Table I). This picture is in disagreement with semilocal DFT results that report $V_{\mathrm{O}}^{\bullet \bullet}$ formation not to cause appreciable distortions in the surface structure [9,11], but it is in line with DFT $+U$ results [10]. The formation of charged $V_{\mathrm{O}}$ was, however, not taken into account in these studies. We find that the singly positively $\left(V_{\mathrm{O}}^{\bullet}\right)$ and doubly positively $\left(V_{\mathrm{O}}^{\mathrm{X}}\right)$ charged vacancies result in structural relaxations similar to $V_{\mathrm{O}}^{\boldsymbol{\bullet \bullet}}$, even though the distance between $\mathrm{Cu}_{\mathrm{CUS}}$ and $\mathrm{Cu}_{\mathrm{NN}}$ is slightly larger compared to $V_{\mathrm{O}}^{\bullet \bullet}$ [see Table I and Fig. 1(c)].

Figure 2(a) illustrates the electronic properties of the stoichiometric nonreconstructed $\mathrm{Cu}_{2} \mathrm{O}$ (111) surface: in agreement with scanning tunneling spectroscopy (STS) $[7,11]$ and photoemission [5] the band gap is significantly smaller than in bulk $\mathrm{Cu}_{2} \mathrm{O}$. This is due to the appearance of a peak at about $1.78 \mathrm{eV}$ from the valence band maximum (VBM) that can be attributed to surface states localized mainly on $\mathrm{Cu}_{\mathrm{CUS}}$ and $\mathrm{Cu}_{\mathrm{CSA}}$ atoms in the outermost $\mathrm{O}-\mathrm{Cu}-\mathrm{O}$ trilayer [see Fig. 2(e)].

On the oxygen deficient reconstructed surface, a $V_{\mathrm{O}}^{\bullet \bullet}$ results in the two excess electrons occupying states resonant with the valence band [VB, see Fig. 2(b)] and in the appearance of an empty localized defect state [DS2 in Fig. 2(b)] below the surface states (about $1.61 \mathrm{eV}$ from VBM). DS2 has contributions of the three $\mathrm{Cu}_{\mathrm{NN}}$ and the three $\mathrm{Cu}_{\mathrm{CUS}}$ atoms closest to the oxygen vacancy [see Fig. 2(f)]. If one oxygen atom and one electron are simultaneously removed to form $V_{\mathrm{O}}^{\bullet}$, the extra electron occupies states resonant with the VB, while an unoccupied defect state appears at $0.17 \mathrm{eV}$, mainly localized on two $\mathrm{Cu}$ atoms far from the $V_{\mathrm{O}}^{\bullet}$ [DS1 in Figs. 2(c) and 2(g)], similar to what we observe for bulk $\mathrm{Cu}_{2} \mathrm{O}$ (SM [20] Fig. S4). Furthermore, in the presence of $V_{\mathrm{O}}^{\bullet}$, DS2 is stabilized and appears at about 1.3-1.4 eV in the gap, while the surface states are pushed up beyond $2.0 \mathrm{eV}$ just below the conduction band (CB). Finally, $V_{\mathrm{O}}^{\mathrm{X}}$ is associated with the empty defect state DS1 merged with the top of the VB and mainly localized on $\mathrm{Cu}$ atoms far away from the defect [see Figs. 2(d) and 2(h)]. The splitting between the DS2 and surface states is still visible, but the corresponding peaks appear at about 1.8 and $2.1 \mathrm{eV}$, respectively, the highest ones being merged with the CB.

Figure 2(i) shows the formation energies of these surface $V_{O}$ in the different charge states as a function of the position of the Fermi energy under O-poor conditions. The neutral defect has a formation energy of about $1.2 \mathrm{eV}$, slightly lower than previously reported using standard DFT functionals (1.4-1.7 eV) [9-11]. As in the bulk (see SM [20] Sec. S2), the neutral defect is most stable, except for Fermi energies just above the VBM, where $V_{\mathrm{O}}^{\mathrm{X}}$ becomes the favored charge state with a thermodynamic transition level $\epsilon(+2 /+$ 1) of $0.20 \mathrm{eV}$. This confirms the existence of doubly charged $V_{\mathrm{O}}^{\mathrm{X}}$ under $p$-type doping, leading to the $(\sqrt{3} \times \sqrt{3})-R 30^{\circ}$ reconstruction. These vacancies strongly repel each other, simple electrostatic point-charge calculations suggesting

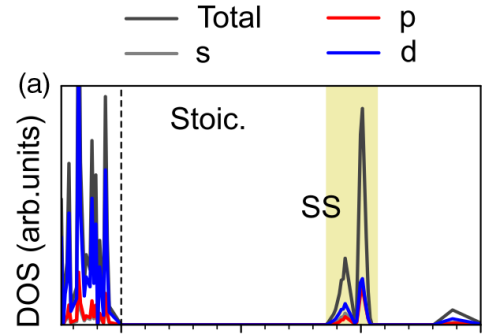

$\odot \mathrm{Cu} \odot \mathrm{O} \mathrm{v}_{\mathrm{O}}$
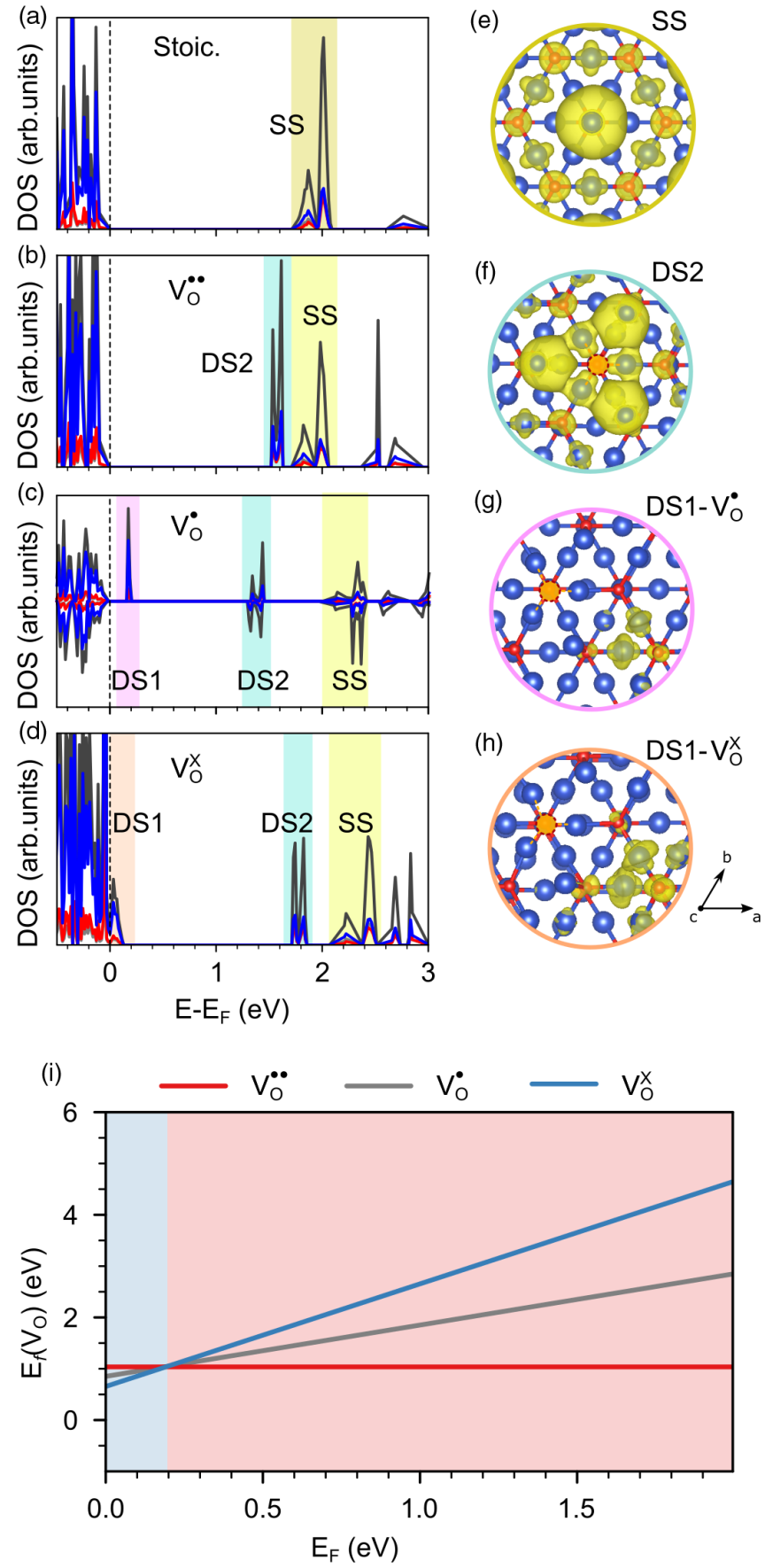

FIG. 2. Density of states for the $(\sqrt{3} \times \sqrt{3}) R 30^{\circ}$ supercell (a) in the stoichiometric case and with one (b) $V_{\mathrm{O}}^{\bullet \bullet}$, (c) $V_{\mathrm{O}}^{\bullet}$, and (d) $V_{\mathrm{O}}^{\mathrm{X}}$. The zero of the energy scale was set at the Fermi energy. For the spin-polarized $V_{\mathrm{O}}^{\bullet}$ calculation, the DOS for the spin-up and spin-down channels are reported with positive and negative values respectively on the $y$ axis. The isosurfaces $\left(2 \times 10^{-2} e / \AA^{3}\right)$ in (e)-(h) correspond to the charge density associated with the defect states (DS) or surface states (SS) highlighted with the corresponding color in plots (a)-(d). (i) Oxygen vacancy formation energy $\left[E_{f}\left(V_{\mathrm{O}}\right)\right]$ under $\mathrm{O}$-poor conditions in different charge states as a function of the Fermi energy ranging from the valence band maximum $\left(E_{F}=0\right)$ up to the experimental band gap for the $\mathrm{Cu}_{2} \mathrm{O}-(111)$ surface. 


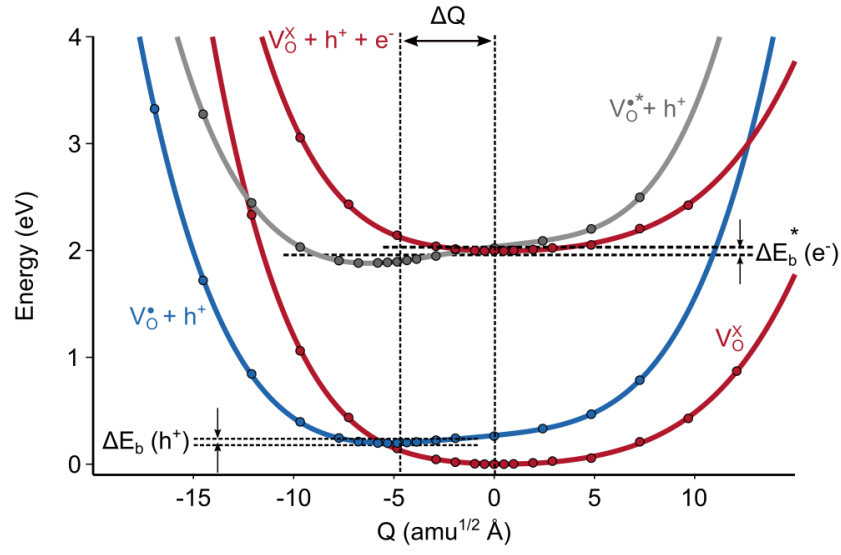

FIG. 3. Configuration coordinate diagram for $V_{\mathrm{O}}(+2 /+1)$ carrier capture at the reconstructed $\mathrm{Cu}_{2} \mathrm{O}-(111)$ surface. The solid circles represent the relative formation energies calculated using hybrid DFT and lines are spline fits. $\Delta E_{\mathrm{b}}$ are carrier capture barriers.

energies larger by $4.5 \mathrm{eV}$ for arrangements other than the ordered reconstruction.

\section{B. Carrier capture}

Bulk calculations performed with better converged parameters compared to previous HSE calculations show that a thermodynamic transition level $\epsilon(0 /+1)$ exists close to the valence band edge (see SM [20] Sec. S3). Bulk oxygen vacancies could therefore, in principle, trap holes, while electron trapping is not possible. The computed hole-capture coefficient (see SM [20] Sec. S4) is, however, very small, demonstrating that neither electron nor hole trapping by bulk $V_{\mathrm{O}}$ is likely.

The calculated 1D configuration coordinate diagram for carrier capture at the reconstructed $(\sqrt{3} \times \sqrt{3})-R 30^{\circ}-(111)$ surface (Fig. 3) shows a very flat potential energy landscape for both the ground and excited states. The ground state is the doubly charged $V_{\mathrm{O}}^{\mathrm{X}}$, while for the excited state we initially consider the singly charged $V_{\mathrm{O}}^{\bullet}+h^{+}$with a hole in the VB. The minima of this ground and excited state are horizontally offset by $\Delta Q=4.83 \mathrm{amu}^{1 / 2} \AA$ (see Table II), due to the large relaxations involving in particular the $\mathrm{Cu}_{\mathrm{CUS}}$ (see Table I), while the energy difference between the two minima is only $0.20 \mathrm{eV}$, corresponding to the $\epsilon(+2 /+1)$ charge transition level. Since all potential energy surfaces in Fig. 3 are anharmonic, we verified that the same adiabatic potential is maintained across the considered range of $Q$ (see SM [20] Sec. S5). The flat landscape and the large lattice relaxations result

TABLE II. Key parameters for carrier capture processes at the reconstructed $\mathrm{Cu}_{2} \mathrm{O}$ (111) surface: total mass-weighted distortions ( $\Delta Q$, in $\left.\mathrm{amu}^{1 / 2} \AA\right)$, ionization energy $(\Delta E$, in $\mathrm{eV})$, carrier capture barrier $\left(\Delta E_{b}\right.$, in eV), carrier capture coefficient $\left(C\right.$, in $\left.\mathrm{cm}^{3} / \mathrm{s}\right)$, and carrier capture cross section $\left(\sigma\right.$ in $\left.\AA^{2}\right)$ at $298 \mathrm{~K}$.

\begin{tabular}{lcccccc}
\hline \hline Defect & Carrier & $\Delta Q$ & $\Delta E$ & $\Delta E_{b}$ & $C$ & $\sigma$ \\
\hline$(+2 /+1)$ & $h^{+}$ & 4.83 & 0.20 & 0.003 & $1.24 \times 10^{-19}$ & $7.87 \times 10^{-11}$ \\
$\left(+2 /+1^{*}\right)$ & $e^{-}$ & 4.83 & 2.00 & 0.011 & $4.87 \times 10^{-9}$ & 3.82 \\
\hline \hline
\end{tabular}

in a small hole capture barrier since the two curves intersect only $0.003 \mathrm{eV}$ above the minimum of $V_{\mathrm{O}}^{\bullet}+h^{+}$. This results in a hole-capture coefficient $C=4.36 \times 10^{-18} \mathrm{~cm}^{3} / \mathrm{s}$ and holecapture cross section $\sigma=2.75 \times 10^{-9} \AA^{2}$ at $298 \mathrm{~K}$ (see SM [20] Fig. S6 for temperature-dependent values). While the potential energy curves of $V_{\mathrm{O}}^{\mathrm{X}}+h^{+}+e^{-}$and $V_{\mathrm{O}}^{\bullet}+h^{+}$intersect, the electron-capture barrier in excess of $2 \mathrm{eV}$ will, given the inverse exponential dependence, result in a very small nonradiative electron capture rate.

Excited states of defects are believed to play a pivotal role in multiphonon emission processes in wide-gap materials [36]. For a $V_{\mathrm{O}}^{\bullet}$ on the reconstructed surface, a spin-conserving excited state is reached by excitation of the extra electron from defect state DS1 to DS2 of the singly charged vacancy [see Fig. 2(c)]. Beyond-DFT methods would, in principle, be necessary to describe excited states, but Alkauskas et al. [36] showed that, for hybrid DFT functionals, accurate results can be obtained by approximating total energy differences by differences in single-particle Kohn-Sham eigenvalues in the spirit of the generalized Koopmans' theorem: at fixed geometry, the total energy of the excited state for a singly charged defect $\left(V_{\mathrm{O}}^{\bullet *}\right)$ is higher than that of the ground state $V_{\mathrm{O}}^{\bullet}$ by the eigenvalue difference between DS2 and DS1 evaluated for the ionized $V_{\mathrm{O}}^{\mathrm{X}}$ state

$$
E_{\mathrm{tot}, V_{\mathrm{O}}^{*}}=E_{\mathrm{tot}, V_{\mathrm{O}}^{*}}+\epsilon_{\mathrm{DS} 2, V_{\mathrm{O}}^{X}}-\epsilon_{\mathrm{DS} 1, V_{\mathrm{O}}^{X}},
$$

where all terms are consistently calculated for the same geometry. We verified by relaxing structures with constrained fractional occupations of defect states as suggested by Ref. [36] that geometry and energy differences for different excited states are negligible and chose to work with the one with a fully occupied DS1. We also verified that for the HSE functional with a mixing parameter of 0.25 , single-particle eigenvalues and total energy differences agree to within $0.1 \mathrm{eV}$. To perform this comparison, Kohn-Sham eigenvalues for charged defective systems have been corrected according to Ref. [47]. As shown in Fig. 3, when this excited state is taken into account, electron capture into $V_{\mathrm{O}}^{* *}$ can occur because the $\left(+2 /+1^{*}\right)$ transition lies closer to the CBM by the intradefect excitation energy of about $2.0 \mathrm{eV}$. The resulting electron-capture barrier of $0.011 \mathrm{eV}$ and the proximity of the excited state to the CB lead to a large capture coefficient $C=$ $3.23 \times 10^{-9} \mathrm{~cm}^{3} / \mathrm{s}$ and cross section $\sigma=2.53 \AA^{2}$ at $298 \mathrm{~K}$ (see SM [20] Fig. S6 for temperature-dependent values). Considering a defect density of $4.58 \times 10^{21} \mathrm{~cm}^{-3}$ (one defect per supercell within $1 \mathrm{~nm}$ from the surface), this electron-capture coefficient results in an electron lifetime in the $\mathrm{CB}$ of about $6.74 \times 10^{-14} \mathrm{~s}$ and a mean free path of $8.61 \times 10^{-07} \mathrm{~cm}$. After the capture process the system can quickly relax from $V_{\mathrm{O}}^{* *}$ to $V_{\mathrm{O}}^{\bullet}$ via intradefect relaxations, the extra electron occupying either DS1 or DS2. This electron-capture process is represented in Fig. 4.

This mechanism for electron capture can be confirmed comparing the theoretical results with the electron dynamics at the (111) surface of $\mathrm{Cu}_{2} \mathrm{O}$ experimentally determined via time-resolved 2PPE [5]. In these experiments, electrons are excited from the VB to the $\mathrm{CB}$ by absorption of a $3-\mathrm{eV}$ laser pulse. The energy distribution of photoexcited electrons above the Fermi energy is monitored by a $6-\mathrm{eV}$ laser pulse as function of the time delay between both pulses. In 


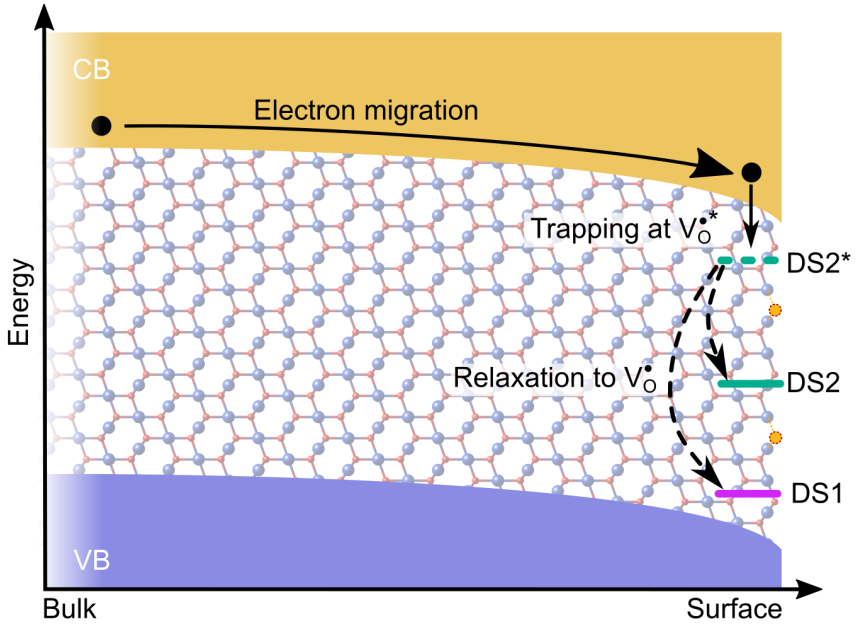

FIG. 4. Schematic representation of electron capture at the reconstructed $(\sqrt{3} \times \sqrt{3}) R 30^{\circ} \mathrm{Cu}_{2} \mathrm{O}-(111)$ surface. Due to surface band bending photoexcited electrons drift towards the surface, where they can either drive photocatalytic reactions (not shown) or be trapped in surface defect states (DS), if available. DS shown in the schematic result from experimentally observed surface oxygen vacancies $\left(V_{\mathrm{O}}\right)$. Direct trapping into DS1 or DS2 is slow, but an electron can very rapidly be trapped into an excited state DS2*, from where it can relax into either DS1 or DS2. Blue and red spheres represents $\mathrm{Cu}$ and $\mathrm{O}$ atoms, respectively, while $\mathrm{V}_{\mathrm{O}}$ are shown in orange.

addition, valence band spectra were measured using ultraviolet photoelectron spectroscopy with $\mathrm{He}_{\alpha}$ radiation $(21.2 \mathrm{eV})$. In Fig. 5 the measured valence and conduction band spectra are shown together with the computed DOS for both surfaces. On the stoichiometric $(1 \times 1)$ surface, the surface and lowlying conduction band states at 1.9 and $2.6 \mathrm{eV}$ above the VBM are present, in good agreement with the computed DOS. The lifetime of excited electrons in the $\mathrm{CB}$ was determined as $10 \mathrm{ps}$. However, for the reconstructed $(\sqrt{3} \times \sqrt{3})-R 30^{\circ}$ surface no photoemission signal from the $\mathrm{CB}$ is observed, but an intense defect state at $0.1 \mathrm{eV}$ and another weaker state at $0.9 \mathrm{eV}$ dominate the spectra. The missing photoemission signal from the CB is evidence for an ultrafast capture of photoexcited electrons by defects on the reconstructed surface. Occupation of those defect states by the excited electrons is faster than filling the low-lying CB states $(0.030 \mathrm{ps})$ in good agreement with the electron lifetime in the $\mathrm{CB}$ predicted by DFT. These defects act as traps, since no noticeable decay of their electron population is observed over a time scale of hundreds of picoseconds. The good agreement between the experimental spectrum obtained in the 2PPE by populating the ground state $V_{\mathrm{O}}^{\mathrm{X}}$ with one extra electron and the computed density of states for the reconstructed surface with $V_{\mathrm{O}}^{\bullet}$ in Fig. 5 confirms the predominance of charged oxygen vacancies in the $(\sqrt{3} \times \sqrt{3})-R 30^{\circ}$ reconstruction of the $\mathrm{Cu}_{2} \mathrm{O}$ (111) surface, as well as the crucial role of the $V_{\mathrm{O}}^{\mathrm{X}} / V_{\mathrm{O}}^{\bullet *}$ transition for the observed efficient electron-capture at this surfaces.

\section{CONCLUSIONS}

In summary, our hybrid DFT results show that bulk defects in $\mathrm{Cu}_{2} \mathrm{O}$ cannot trap electrons and are highly inefficient hole
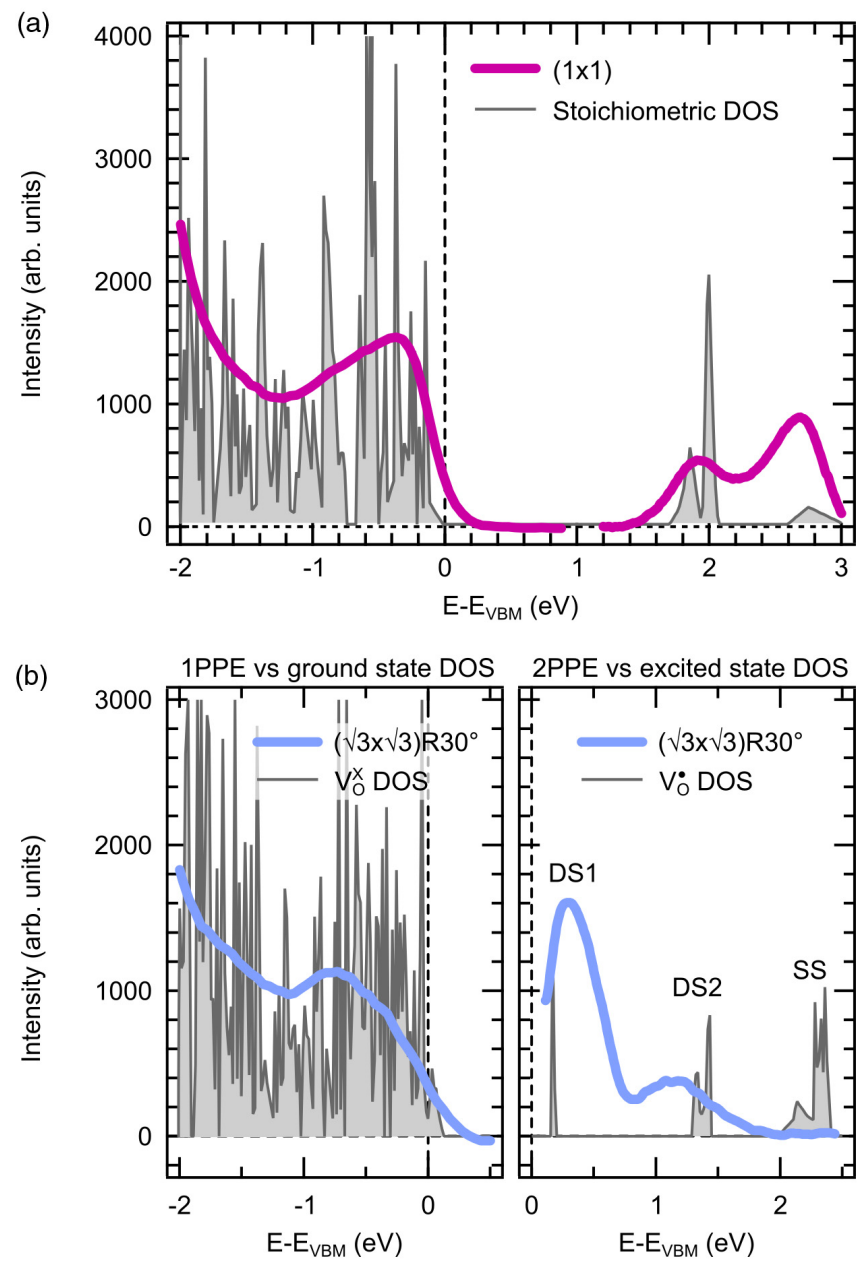

FIG. 5. Comparison of the experimentally measured (dashed lines) and computed (solid lines) density of states for (a) the stoichiometric $(1 \times 1)$ and $(\mathrm{b})$ the reconstructed $(\sqrt{3} \times \sqrt{3})-R 30^{\circ}-(111)$ $\mathrm{Cu}_{2} \mathrm{O}$ surface with $V_{\mathrm{O}}$. For the valence band, determined using 1PPE, we compare with the ground-state $V_{\mathrm{O}}^{\mathrm{X}}$ DOS, whereas for the empty states, determined using 2PPE, we compare with the excited-state $V_{\mathrm{O}}^{\bullet}$ DOS. For the spin-polarized $V_{\mathrm{O}}^{\bullet}$ DOS, the spin-up and spin-down channels have been summed. Note the missing photoelectron signals at energies higher than $2 \mathrm{eV}$ in (b).

traps. We further confirm that the $\sqrt{3} \times \sqrt{3}$ )- $R 30^{\circ}$ reconstruction experimentally observed under oxygen-poor conditions on the $\mathrm{Cu}_{2} \mathrm{O}$ (111) surface is associated with a $1 / 3$ monolayer of charged oxygen vacancies, as previously hypothesized based on experiments. We report an excellent agreement between experimental spectra and computed densities of states that confirm the singly charged $\left(V_{\mathrm{O}}^{\bullet}\right)$ state of these defects after electron trapping, and hence support the computed doubly charged $V_{\mathrm{O}}^{\mathrm{X}}$ ground state. Due to electrostatic repulsion, these defects form a highly ordered structure that strongly suppresses electron transport. In particular, the excited state of the $V_{\mathrm{O}}^{\bullet}$ plays a crucial role in efficient electron capture at the surface. Electrons are trapped by a transition from $V_{\mathrm{O}}^{\mathrm{X}}$ to the excited state $V_{\mathrm{O}}^{\bullet *}$. After the capture process, the system can relax to $V_{\mathrm{O}}^{\bullet}$ via intradefect relaxation, the electron finally being trapped in the defect states associated with the singly charged 
defect. This process is predicted to have a capture coefficient of about $10^{-9} \mathrm{~cm}^{3} / \mathrm{s}$ leading to a free-carrier lifetime of $0.04 \mathrm{ps}$, in good agreement with experimental observations. The findings demonstrate the predominance of surface oxygen vacancies in limiting the photocurrent of $\mathrm{Cu}_{2} \mathrm{O}$ based heterostructures and provide a solid basis for the engineering of more efficient $\mathrm{Cu}_{2} \mathrm{O}$ photoelectrodes.

Data associated with the calculations are available on the Materials Cloud [48].

\section{ACKNOWLEDGMENTS}

This research was supported by the NCCR MARVEL, funded by the Swiss National Science Foundation and by the Swiss National Science Foundation (Grants No. 200020_172641, No. PP00P2_157615, and No. PP00P2_187185). Computational resources for this work were provided by the University of Bern (on the HPC cluster UBELIX, http://www.id.unibe.ch/hpc) and by the Swiss National Supercomputing Center (CSCS) under Project IDs No. s955 and No. s1033.
[1] B. K. Meyer, A. Polity, D. Reppin, M. Becker, P. Hering, P. J. Klar, T. Sander, C. Reindl, J. Benz, M. Eickhoff, C. Heiliger, M. Heinemann, J. Bläsing, A. Krost, S. Shokovets, C. Müller, and C. Ronning, Binary copper oxide semiconductors: From materials towards devices, Phys. Status Solidi B 249, 1487 (2012).

[2] W. Niu, T. Moehl, W. Cui, R. Wick-Joliat, L. Zhu, and S. D. Tilley, Extended light harvesting with dual $\mathrm{Cu}_{2} \mathrm{O}$-based photocathodes for high efficiency water splitting, Adv. Energy Mater. 8, 1702323 (2018).

[3] L. Pan, J. H. Kim, M. T. Mayer, M.-K. Son, A. Ummadisingu, J. S. Lee, A. Hagfeldt, J. Luo, and M. Grätzel, Boosting the performance of $\mathrm{Cu}_{2} \mathrm{O}$ photocathodes for unassisted solar water splitting devices, Nat. Catal. 1, 412 (2018).

[4] M. Borgwardt, S. Omelchenko, M. Favaro, P. Plate, C. Höhn, D. Abou-Ras, K. Schwarzburg, R. Van de Krol, H. Atwater, N. Lewis, R. Eichberger, and D. Friedrich, Femtosecond timeresolved two-photon photoemission studies of ultrafast carrier relaxation in $\mathrm{Cu}_{2} \mathrm{O}$ photoelectrodes, Nat. Commun. 10, 2106 (2019).

[5] L. Grad, Z. Novotny, M. Hengsberger, and J. Osterwalder, Influence of surface defect density on the ultrafast hot carrier relaxation and transport in $\mathrm{Cu}_{2} \mathrm{O}$ photoelectrodes, Sci. Rep. 10, 10686 (2020).

[6] K. H. Schulz and D. F. Cox, Photoemission and low- energyelectron-diffraction study of clean and oxygen- dosed $\mathrm{Cu}_{2} \mathrm{O}$ (111) and (100) Surfaces, Phys. Rev. B 43, 1610 (1991).

[7] A. Önsten, M. Göthelid, and U. O. Karlsson, Atomic structure of $\mathrm{Cu}_{2} \mathrm{O}(111)$, Surf. Sci. 603, 257 (2009).

[8] A. Önsten, J. Weissenrieder, D. Stoltz, S. Yu, M. Göthelid, and U. O. Karlsson, Role of defects in surface chemistry on $\mathrm{Cu}_{2} \mathrm{O}(111)$, J. Phys. Chem. C 117, 19357 (2013).

[9] B.-Z. Sun, W.-K. Chen, J.-D. Zheng, and C.-H. Lu, Roles of oxygen vacancy in the adsorption properties of $\mathrm{Co}$ and $\mathrm{NO}$ on $\mathrm{Cu}_{2} \mathrm{O}(111)$ surface: Results of a first-principles study, Appl. Surf. Sci. 255, 3141 (2008).

[10] L. I. Bendavid and E. A. Carter, First-principles predictions of the structure, stability, and photocatalytic potential of $\mathrm{Cu}_{2} \mathrm{O}$ surfaces, J. Phys. Chem. B 117, 15750 (2013).

[11] R. Zhang, L. Li, L. Frazer, K. B. Chang, K. R. Poeppelmeier, M. K. Y. Chan, and J. R. Guest, Atomistic determination of the surface structure of $\mathrm{Cu}_{2} \mathrm{O}(111)$ : Experiment and theory, Phys. Chem. Chem. Phys. 20, 27456 (2018).

[12] X. Yu, C. Zhao, T. Zhang, and Z. Liu, Molecular and dissociative $\mathrm{O}_{2}$ adsorption on the $\mathrm{Cu}_{2} \mathrm{O}(111)$ Surface, Phys. Chem. Chem. Phys. 20, 20352 (2018).
[13] A. Gloystein, N. Nilius, J. Goniakowski, and C. Noguera, Nanopyramidal reconstruction of $\mathrm{Cu}_{2} \mathrm{O}(111)$ : A Long- standing surface puzzle solved by STM and DFT, J. Phys. Chem.C 124, 26937 (2020).

[14] S. Koirala, M. Takahata, Y. Hazama, N. Naka, and K. Tanaka, Relaxation of localized excitons by phonon emission at oxygen vacancies in $\mathrm{Cu}_{2} \mathrm{O}$, J. Lumin. 155, 65 (2014).

[15] M. Nolan and S. D. Elliott, The $p$-type conduction mechanism in $\mathrm{Cu}_{2} \mathrm{O}$ : A first principles study, Phys. Chem. Chem. Phys. 8 5350 (2006).

[16] H. Raebiger, S. Lany, and A. Zunger, Origins of the p-type nature and cation deficiency in $\mathrm{Cu}_{2} \mathrm{O}$ and related materials, Phys. Rev. B 76, 045209 (2007).

[17] M. Nolan, Defects in $\mathrm{Cu}_{2} \mathrm{O}, \mathrm{CuAlO}_{2}$ and $\mathrm{SrCu}_{2} \mathrm{O}_{2}$ transparent conducting oxides, Thin Solid Films 516, 8130 (2008).

[18] D. O. Scanlon and G. W. Watson, Undoped $n$-type $\mathrm{Cu}_{2} \mathrm{O}$ : Fact or fiction? J. Phys. Chem. Lett. 1, 2582 (2010).

[19] B. Huang, Intrinsic deep hole trap levels in $\mathrm{Cu}_{2} \mathrm{O}$ with selfconsistent repulsive coulomb energy, Solid State Commun. 230, 49 (2016).

[20] See Supplemental Material at http://link.aps.org/supplemental/ 10.1103/PhysRevResearch.3.043219 for method details, bulk $\mathrm{Cu}_{2} \mathrm{O}$ results, and additional surface calculations.

[21] D. O. Scanlon, B. J. Morgan, G. W. Watson, and A. Walsh, Acceptor Levels in $p$-Type $\mathrm{Cu}_{2} \mathrm{O}$ : Rationalizing Theory and Experiment, Phys. Rev. Lett. 103, 096405 (2009).

[22] G. Kresse and J. Hafner, Ab initio molecular dynamics for liquid metals, Phys. Rev. B 47, 558(R) (1993).

[23] G. Kresse and J. Hafner, Ab initio molecular- dynamics simulation of the liquid-metalamorphous- semiconductor transition in germanium, Phys. Rev. B 49, 14251 (1994).

[24] G. Kresse and J. Furthmüller, Efficiency of ab-initio total energy calculations for metals and semiconductors using a plane-wave basis set, Comput. Mater. Sci. 6, 15 (1996).

[25] G. Kresse and J. Furthmüller, Efficient iterative schemes for $a b$ initio total-energy calculations using a plane-wave basis set, Phys. Rev. B 54, 11169 (1996).

[26] J. Heyd, G. E. Scuseria, and M. Ernzerhof, Hybrid functionals based on a screened coulomb potential, J. Chem. Phys. 118 8207 (2003).

[27] J. Heyd and G. E. Scuseria, Efficient hybrid density functional calculations in solids: Assessment of the Heyd-ScuseriaErnzerhof screened coulomb hybrid functional, J. Chem. Phys. 121, 1187 (2004).

[28] P. E. Blöchl, Projector augmented-wave method, Phys. Rev. B 50, 17953 (1994). 
[29] G. Kresse and D. Joubert, From ultrasoft pseudopotentials to the projector augmented-wave method, Phys. Rev. B 59, 1758 (1999).

[30] C. Freysoldt, B. Grabowski, T. Hickel, J. Neugebauer, G. Kresse, A. Janotti, and C. G. Van de Walle, First- principles calculations for point defects in solids, Rev. Mod. Phys. 86, 253 (2014).

[31] S. Lany and A. Zunger, Assessment of correction methods for the band-gap problem and for finite-size effects in supercell defect calculations: Case studies for $\mathrm{ZnO}$ and GaAs, Phys. Rev. B 78, 235104 (2008).

[32] C. Freysoldt and J. Neugebauer, First-principles calculations for charged defects at surfaces, interfaces, and two-dimensional materials in the presence of electric fields, Phys. Rev. B 97, 205425 (2018).

[33] K. Huang and A. Rhys, Theory of light absorption and nonradiative transitions in F-centres, Proc. $\mathrm{R}$. Soc. London Ser. A 204, 406 (1950).

[34] C. H. Henry and D. V. Lang, Nonradiative capture and recombination by multiphonon emission in GaAs and GaP, Phys. Rev. B 15, 989 (1977).

[35] A. Alkauskas, Q. Yan, and C. G. Van de Walle, First-principles theory of nonradiative carrier capture via multiphonon emission, Phys. Rev. B 90, 075202 (2014).

[36] A. Alkauskas, C. E. Dreyer, J. L. Lyons, and C. G. Van de Walle, Role of excited states in shockley-read-hall recombination in wide-band-gap semiconductors, Phys. Rev. B 93, 201304(R) (2016).

[37] H. Huang, Adiabatic approximation theory and static coupling theory of nonradiative transition, Sci. Sin. 24, 27 (1981).
[38] S. Kim, S. N. Hood, and A. Walsh, Anharmonic lattice relaxation during nonradiative carrier capture, Phys. Rev. B 100, 041202(R) (2019).

[39] A. Alkauskas, J. L. Lyons, D. Steiauf, and C. G. Van de Walle, First-Principles Calculations of Luminescence Spectrum Line Shapes for Defects in Semiconductors: The Example of GaN and ZnO, Phys. Rev. Lett. 109, 267401 (2012).

[40] K. Bystrom, D. Broberg, S. Dwaraknath, K. A. Persson, and M. Asta, Pawpyseed: Perturbation-extrapolation band shifting corrections for point defect calculations, arXiv:1904.11572.

[41] S. Kim, J.-S. Park, S. N. Hood, and A. Walsh, Lone-pair effect on carrier capture in $\mathrm{Cu}_{2} \mathrm{ZnSnS}_{4}$ solar cells, J. Mater. Chem. A 7, 2686 (2019).

[42] S. Kim, J. A. Márquez, T. Unold, and A. Walsh, Upper limit to the photovoltaic efficiency of imperfect crystals from first principles, Energy Environ. Sci. 13, 1481 (2020).

[43] A. Fonari and C. Sutton, Effective mass calculator (2012), https: //github.com/afonari/emc.

[44] L. Razinkovas, M. W. Doherty, N. B. Manson, C. G. V. de Walle, and A. Alkauskas, Vibrational and vibronic structure of isolated point defects: The nitrogen-vacancy center in diamond, Phys. Rev. B 104, 045303 (2021).

[45] S. Kim, N. S. Hood, and A. Walsh, Carriercapture, https:// github.com/WMD-group/CarrierCapture.jl (2019).

[46] F. A. Kröger and H. J. Vink, Relations between the concentrations of imperfections in crystalline solids, Solid State Phys. 3, 307 (1956)

[47] W. Chen and A. Pasquarello, Correspondence of defect energy levels in hybrid density functional theory and many-body perturbation theory, Phys. Rev. B 88, 115104 (2013).

[48] https://doi.org/10.24435/materialscloud:rr-2n. 